\title{
Movilidad y cooperación internacional en la formación de docentes indígenas interculturales
}

\section{Mobility and international cooperation in the educational formation of intercultural indigenous teachers \\ - Adán Hernández \\ Escuela Normal Intercultural Lic. Manuel Larraínzar, México \\ Fecha de recepción: 31 de marzo de 2019 \\ Fecha de aprobación: 9 de mayo de 2019 \\ DOI: http://dx.doi.org/10.15304/ricd.3.10.5938}

\section{NOTAS BIOGRÁFICAS}

Adán Hernández es profesor investigador en la Escuela Normal Intercultural "Lic. Manuel Larraínzar" (Chiapas, México) y doctor en Estudios Regionales por la Universidad Autónoma de Chiapas (UNACH).

Contacto: adanhm60@hotmail.com

\section{Resumen}

En este artículo se discuten avances de investigación sobre las experiencias de movilidad y cooperación internacional de alumnos indígenas que se forman como profesores en las escuelas normales rurales e interculturales de Chiapas. El objetivo fue identificar la participación de los alumnos en los programas y reconstruir las experiencias de aprendizaje que valoran para la formación como docentes indígenas.

Se aplicó un estudio cualitativo, con entrevistas en profundidad para recuperar las experiencias. Los resultados dan cuenta de la brecha que aún existe en el ingreso de alumnos indígenas a las escuelas normales interculturales, así como la escasa, casi nula participación en los programas de movilidad. Las experiencias de los alumnos en estos programas dan cuenta de aprendizajes, competencias profesionales, el valor de la interculturalidad, así como los retos que impone la internacionalización de la educación superior a la formación docente.

\section{Abstract}

In this article we discuss advances in academic research about the experiences of school mobility and international cooperation of indigenous students who are trained as teachers in rural and intercultural schools in Chiapas. The purpose was to identify the participation of students in the programs and rebuild the learning experiences that they value for training as indigenous teachers.

A qualitative study was applied, with in-depth interviews to recover the experiences. The results show the gap that still exists in the entrance of indigenous students to intercultural schools, as well as the scarce, there das very little particopation in the mobility programs. The experiences of students in these programs, account for learning, professional skills, the value of interculturality, as well as the challenges imposed by the internationalization of higher education to teacher training.

\section{Palabras clave}

Internacionalización, interculturalidad, docencia, movilidad, educación indígena. 


\section{Sumario}

1. Introducción

2. Planteamiento del problema

3. Cooperación y movilidad internacional en la formación de docentes indígenas interculturales

4. La educación intercultural, más allá de lo conceptual

5. Metodología

6. Resultados

6.1 Estadísticas preocupantes sobre la cooperación y movilidad internacional

6.2 Experiencias de movilidad y cooperación internacional desde los actores

7. Conclusiones

\section{Contents}

1. Introduction

2. Problem research

3. International school cooperation and mobility in the teaching training of intercultural indigenous teachers

4. Intercultural education, beyond the conceptual

5. Methodology

6. Results

6.1 Troubling statistics on international cooperation and mobility

6.2 Experiences of school mobility and international cooperation from the actors

7. Conclusions 


\section{INTRODUCCIÓN}

La constante tensión entre la política educativa nacional y la exigencia de una educación propia propuesta desde las comunidades indígenas, propicia profundas discusiones con relación a lo intercultural en el ámbito educativo. Puntualmente se señalan aquellas políticas educativas que responden al cultural slogan, que esencializa lo indígena e invisibiliza las propuestas de las comunidades indígenas. Aunado a esto, se observa una política de internacionalización -imposible negar la influencia que ésta genera en los estudiantes universitarios de origen indígena que se integran a los procesosque nuclea lo propio con la diversidad existente en los contextos sociales.

La educación intercultural en México se impulsó como una propuesta educativa con distintos ejes, entre los que destacan la creación de políticas, planes y programas de estudios en universidades y escuelas normales a lo largo y ancho del país, priorizando en todo momento los contextos con mayor población indígena. Esta tendencia educativa puntualizó la educación intercultural como eje articulador de la educación indígena con especial atención al rescate del "conocimiento, la lengua y la cultura" de las comunidades indígenas.

Para el caso de las Escuelas Normales en el 2004 se instrumentó una política de reforma curricular que posibilitó la creación del Plan de Estudios de la Licenciatura en Educación Preescolar y Primaria Intercultural Bilingüe (LEPIB), y la conversión de Escuelas Normales Generales a Escuelas Normales Interculturales. La propuesta educativa intercultural se propuso la formación de profesores interculturales bilingües que atendieran la educación preescolar y primaria de las comunidades indígenas a lo largo y ancho del país, en respuesta a ello se crearon 24 Escuelas Normales Interculturales (SEP-DGESPE, 2005).

En el caso de Chiapas, México, se reformaron los Planes de Estudios de tres escuelas normales: Escuela Normal Indígena Intercultural “Jacinto Canek", Escuela Normal Primaria "Lic. Manuel Larraínzar" y la Escuela Normal del Estado, en las que se implementó la Licenciatura en Educación Intercultural Bilingüe. Es importante mencionar, que en la republica mexicana en las que se crearon las Escuelas Normales Interculturales es visible la diferenciación entre aquellas que son indígenas interculturales, y las que únicamente se asumen como interculturales. Esta diversificación tiene que ver con un posicionamiento ideológico, político e intelectual que entreteje una diversidad de significados de lo que se entiende por educación intercultural bilingüe o educación indígena intercultural; la arena discursiva se torna compleja en tanto lo indígena se asume como política educativa asimilatoria, integracionista o esencialista, sin recuperar una propuesta educativa propia de las comunidades indígenas, en el que el empoderamiento es necesario.

La educación intercultural no puede entenderse exclusivamente como proyecto político institucional, mucho menos implementarse como propuesta didáctica romántica-esencialista en los espacios escolares. Por el contra-rio se requiere posibilitar un análisis profundo sobre las necesidades educativas que demandan los contextos en el que se enmarcan. Urge dimensionar el análisis hacia los desafíos de una propuesta epistémica, pedagógica, filosófica y política que posibilite afrontar los retos educativos reconociendo incertidumbres y complejidades en un contexto de globalización e internacionalización de la educación, en el que lo intercultural emerge como una condición ineludible de mediación entre lo global y lo local.

En la dialéctica relacional local-global, la educación intercultural tensa la función de las instituciones educativas (especialmente en instituciones de educación superior que en la actualidad se integran a los proyectos de internacionalización), en correspondencia con las transformaciones sociales y el constante contacto cultural planetario-global. Se asumen estrategias que deben replantearse no únicamente como agendas de geopolítica-educativa, sino en la integración plural del reconocimiento de lo local como emergencia, la revaloración y apropiación étnica y la educación propia y popular. Estas últimas encarnan prácticas realmente alternativas y fecundadoras de la vida en el sentido pleno de su diversidad irreductible (Gómez, 2014). Como puntualiza Navarrete (2016) discutir aspectos como

la erosión de la autonomía [...] de las univer-sidades; la movilidad internacional [...]; los valo-res planetarios y los valores nacionales; los rankings y el modelo universitario transnacio-nal; el impacto de la economía global en la proble-mática educativa local; la educación de las migraciones transfronterizas. (p. 13).

La educación superior impone el reto de elaborar una nueva narrativa social e individual, volver a escribir el discurso acerca de la educación intercultural a la luz de nuevas condiciones sociales y experiencias individuales del contexto 
que nos toca vivir. En correspondencia, se asume que es difícil negar los procesos de internalización en el que actualmente se integran los jóvenes indígenas que se forman en las escuelas normales interculturales para ser profesores. Sin embargo, es necesario distanciarse para no caer en el slogan de la interculturalidad funcional que "continúa su perspectiva multiculturalista a partir de la cual el diálogo solo es posible en el contexto de globalización mundial" (Gómez, 2014, p. 128), en el que se puntualizan a manera de escolarización una serie de competencias que los docentes deben realizar para recuperar la lengua, la cultura y la identidad como mera reproducción. Sin que se reconozca la permanente recreación de la cultura local como potenciador, también, de la dinámica social.

\section{PLANTEAMIENTO DEL PROBLEMA}

En el programa de movilidad internacional propuesta desde el Plan de Desarrollo 20132018, se asumió promover la movilidad de alumnos de educación superior que cursan estudios de grado en carreras que conducen al ejercicio de la profesión docente, a través de diferentes experiencias en contextos internacionales. Lo que permitió que en 2015, 135 estudiantes y profesores vivieran las primeras experiencias de cooperación internacional en países como Francia y España (DGESPE, 2018).

Aunque en 2014 se firma el Acuerdo Específico para el Financiamiento del Proyecto Paulo Freire de Movilidad Académica para Estudiantes de Programas Universitarios de Formación del Profesorado, así como en los Acuerdos tomados en la Declaración de la XXIV Conferencia Iberoamericana de Ministros de Educación, con la Secretaría de Educación Pública (SEP), a través de la Subsecretaría de Educación Superior (SES) y de la Dirección General de Educación Superior para Profesionales de la Educación (DGESPE), hasta 2016 se logró que 64 estudiantes participaran en este proyecto, que se suman a 87 estudiantes y directores a Francia y 145 con movilidad nacional.

En efecto, sorprendentemente como sostiene Arriaga, Cabrera y Enríquez (2018), para el caso del Proyecto Paulo Freire entre 2016 y 2017 , 100 estudiantes, representantes de las 32 entidades federativas del país, y de 60 escuelas normales, realizaron estancias académicas en 9 países de la región, pero al mismo tiempo el proyecto posibilitó que 39 universitarios provenientes de 6 países: Chile, Cuba, Ecuador, Pana- má, Paraguay y Uruguay vivieran experiencias educativas en las Instituciones de Educación Superior (IES) del contexto mexicano.

El impacto de los proyectos de apoyo propuestos desde la DGESPE para escuelas normales, han sido cada vez más sustanciales, diversificando las condiciones de participación de los actores educativos de estas instituciones, alcanzando porcentajes importantes en todas las escuelas normales del país; al respecto entre 2015-2018, 1,326 alumnos, docentes, directivos, y autoridades educativas participaron en los programas de movilidad nacional e internacional, destacando países como España, Francia y Canadá, además de Iberoamérica. La estrategia que se asumió, para integrar cada vez más a los estudiantes de las escuelas normales, fue a partir de convocatorias nacionales. El principio fundamental fue la inclusión y diversificación del apoyo a quienes cumplieran con los requisitos propuestos.

El escenario, en el que se están generando las condiciones para el fortalecimiento del programa de movilidad, es resultado de la cooperación entre distintas instancias gubernamentales y educativas, asociaciones profesionales, representantes del sector productivo y organismos de diversa índole con una manifiesta perocupación por la internacionalización de la educación superior. De las experiencias de movilidad y cooperación internacional de estudiantes de escuelas normales (producto de los proyectos propuestos por la DGESPE) es necesario analizar el impacto que se está generando en la formación inicial de docentes indígenas interculturales, así como los procesos de formación pertinentes. Partiendo del supuesto básico de que las IES juegan un papel importante en un escenario nacional e internacional que favorece el desarrollo económico, social, cultural de los países que generan estos proyectos.

En el caso de Chiapas, espacio geográfico en el que desarrolló este proyecto, han sido beneficiados 7 estudiantes indígenas para movilidad internacional de tres escuelas normales: 6 de la Escuela Normal Mactumactzá, de Tuxtla Gutiérrez, en el proyecto México-España, y 1 alumna de la Escuela Normal Primaria “Lic. Manuel Larraínzar", de San Cristóbal de Las Casas, que participó en el programa de movilidad MéxicoCanadá.

También se logró la movilidad de 6 estudiantes en el proyecto de movilidad interna o naciónal: 4 alumnos de la Escuela Normal Mactumactzá, en Tuxtla Gutiérrez, que se movilizaron a la Benemérita Escuela Normal "M. F. Martínez", 
en Monterrey; así como 2 alumnos de la Escuela Normal Experimental, en San Cristóbal de Las Casas, de estos, 1 alumna en la Universidad Autónoma de Baja California y 1 alumno a la Escuela Normal de San Luis Potosí. En total se han beneficiado tres escuelas normales con un total de 13 alumnos en los proyectos de movilidad y cooperación internacional y nacional.

En la investigación ha interesado conocer las experiencias de formación de estos alumnos en los contextos nacionales e internacionales. Se ha hecho énfasis en identificar:

-¿cuál es el total de alumnos indígenas del Estado de Chiapas que han participado en los proyectos de movilidad nacional e internacional?

-¿cuáles son las experiencias de aprendizaje recuperadas durante la movilidad y cooperación internacional en otros contextos como alumnos indígenas?

- ¿cómo ha beneficiado el proyecto de movilidad en la formación de docentes interculturales indígenas?

-¿cuáles son las competencias docentes que se han fortalecido a partir de los proyectos de movilidad?

- ¿qué retos demanda la formación inicial docente intercultural en las Escuelas Normales de Chiapas con relación a las experiencias internacionales?

\section{COOPERACIÓN Y MOVILIDAD INTERNACIONAL EN LA FORMACIÓN DE DOCENTES INDÍGENAS INTERCULTURALES}

El proceso de internacionalización de la Educación Superior (producto de los procesos globales) ha propiciado la transformación, no sólo de las instituciones educativas, sino que al tiempo obliga a reconfigurar las políticas internacionales, nacionales y regionales para favorecer programas de cooperación en los procesos de formación, especialización, investigación, difusión y vinculación.

Desde 1998, la Organización de las Naciones Unidas para la Educación, la Ciencia y la Cultura (UNESCO) centró la discusión en los desafíos para la educación superior como prioridad para el cambio y desarrollo de la educación a partir de una serie de estrategias para que profesores y estudiantes lograran establecer los vínculos que exigen las demandas sociales, académicas y culturales.
La Asociación Nacional de Universidades e Instituciones de Educación Superior (ANUIES, 2019) sostiene que la internacionalización se ha convertido en un elemento estratégico para el fortalecimiento de las instituciones de educación superior a través del aprovechamiento de las ventajas que surgen de la cooperación académica.

Lo que favorece el proceso continuo de transformación integral de las instituciones, que incluye la docencia, la investigación, la difusión de la cultura y la extensión de los servicios.

Basado en los conceptos clave de calidad, pertinencia, equidad y eficiencia, orientado a la incorporación de contenidos, materiales y actividades de cooperación, colaboración y compromiso internacionales.

Que prepare a los estudiantes para desempeñarse con eficacia en un mundo interdependiente como profesionales con formación humanística y axiológica, versátiles y dinámicos con capacidad de autoaprendizaje, comprometidos con la problemática mundial y que aprecien y valoren la diversidad cultural.

Un aspecto central que se sostiene con respecto al proceso de internacionalización de la educación superior hace referencia a un proceso en el cual la dimensión internacional se incorpora a ella en sus aspectos culturales y estratégicos, así como en sus funciones de formación, investigación y extensión; situación que se refleja en la oferta ofrecida y en las capacidades institucionales (Sebastián, 2004).

Para Hudzik (2015) la internacionalización, si bien se manifiesta como necesidad global, su principal apoyatura está en lograr una educación integral, que no solo impacta en las instituciones, sino que posibilita alianzas y relaciones en distintos ámbitos académicos.

Con estas premisas, las IES han reconfigurado sus funciones, dinamizado sus procesos internos y externos, para establecer políticas que favorezcan estos procesos de internacionalización en los que la competitividad y capacidad académica son detonadores en el ámbito internacional.

A este escenario se integran las escuelas normales del país que, desde 1984, han estado directamente vinculadas a las funciones sustantivas que corresponden a las IES.

Destacan aquellas que refieren a la docencia, desarrollo de investigación, gestión y vinculación y tutoría, como parte del fortalecimiento de la capacidad académica. 
También integró programas de formación y capacitación de los docentes de las escuelas normales, a partir de las acciones directas de la DGESPE, que desde 2001 hasta 2018 implementó programas de transformación de las escuelas normales, centrando la atención en los planes y programas de estudios, la capacidad y competitividad académica y la gestión institucional.

El esquema de apoyo de la DGESPE para fortalecer las escuelas normales fue a partir de los programas siguientes: Programa de Transformación y Fortalecimiento Académico de las Escuelas Normales (PTFAEN); Programa de Mejoramiento Institucional de las Escuelas Normales Públicas (PROMIN); Programa de Fortalecimiento de la Calidad en Instituciones Educativas (PROFOCIE); Plan de Apoyo a la Calidad Educativa y la Transformación de las Escuelas Normales (PACTEN). Este último centrando los esfuerzos en garantizar lo establecido en el Plan Nacional de Desarrollo 2013-2018, que intenta garantizar una educación para la adquisición de competencias que sirvan a lo largo de la vida, producto de la exigencia de internacionalización de la educación a nivel global.

Con las estrategias de fortalecimiento propuestas para las escuelas normales, aparecen los programas de movilidad académica. De acuerdo con Barnett (2013) movilidad e internacionalización están estrechamente ligados, ya que la movilidad busca establecer los vínculos más allá de las fronteras nacionales con relación en relación al conocimiento, la ciencia y la cultura.

En este caso, el propósito central del Programa Nacional de Movilidad Académica para la Educación Superior es impulsar y fortalecer la internacionalización; dimensión a la que deben estar integradas las escuelas normales y las IES, y conlleva acciones como: el establecimiento de redes internacionales de cooperación en las funciones sustantivas, el intercambio académico, la movilidad estudiantil y docente o el establecimiento de sistemas de acreditación de estudios.

En el marco de la internacionalización de la educación superior, más allá de las visiones utilitaristas en el fortalecimiento de la formación profesional, como afirma Wadhwa (2016) se requiere pensar la internacionalización como un proyecto integral de largo aliento que rebase una educación superior caracterizada por inte-reses económicos, específicamente por maximi-zar utilidades y capturar el mercado estudiantil en otros países.
Ello implica enmarcar la política educativa nacional para que asuma la formación en las IES: acorde con el mundo, en el devenir de las necesidades sociales. De este modo, la internacionalización busca articular un compromiso multilateral y al eliminar las fronteras de formación impulsa redes de colaboración, el desarroIlo integral, la mejora sustantiva en las actividades de los actores educativos y el desarrollo de proyectos en comunidad.

Esta relación, que se está acentuado entre internacionalización y movilidad, tiene la intención de aclarar cómo se está comprendiendo la movilidad en las escuelas normales. Como refiere Montes (2018) en este caso:

los programas de movilidad nacional implementados por la DGESPE para las escuelas normales tienen como objetivo principal fortalecer la formación docente y disciplinaria de los estudiantes. Adicionalmente, en los programas de movilidad internacional de estudiantes se trata de perfeccionar el dominio de lenguas extranjeras y conocer los sistemas educativos, especialmente los relacionados con la formación docente (p. 3).

Esto implica que también se reorganizó la estructura curricular, flexibilizando los procesos de formación y acreditación de los estudiantes que deciden participar en las estancias académicas en su localidad, región, país e internacional con sus homólogas en formación y con universidades nacionales o internacionales.

Aunque las políticas de movilidad para las escuelas normales acentúan el fortalecimiento de la formación inicial docente con estudiantes, también se ha propuesto que directivos y profesores se integren a estas actividades; algunos programas de movilidad se han generado a partir de las becas de DGESPE, pero también existen experiencias de profesores que se han integrado a la movilidad con los beneficios del Programa de Desarrollo Profesional Docente (PRODEP) a través de los Cuerpos Académicos, y de los profesores becados por parte del Consejo Nacional de Ciencia y Tecnología (CONACyT).

\section{$Y$ aunque:}

la movilidad académica suele estar situada dentro de un esquema de trabajo interinstitucional, nacional o internacional, es posible que el flujo de personas se desarrolle a partir de la iniciativa de los propios sujetos o como parte de proyectos específicos que no necesariamente involucran el trabajo con varias IES o centros de investigación. (García, 2013, p. 62).

En este caso, la investigación buscó recuperar la experiencia de estudiantes en el programa de movilidad nacional e internacional, ya que 
desde la propuesta de fortalecimiento de las escuelas normales los programas de movilidad estudiantil ofrecen a los jóvenes la posibilidad de desempeñarse competentemente a escala nacional e internacional, con alto grado de adaptación y creatividad en el mundo laboral, conscientes de su responsabilidad social y con amplias capacidades para vivir y desarrollarse en un entorno global y multicultural.

La DGESPE desde 2016 a 2018 ha integrado una política de movilidad académica a partir de cuatro programas que han permitido a las escuelas normales estar en el marco de la internalización de sus procesos académicos con calidad y pertinencia:

- El Programa de Capacitación de Directores de Escuelas Normales en Francia, desarrollado en la Escuela Superior de la Educación Nacional, con sede en Poitiers, Francia, con la finalidad de formar en gestión educativa a directores de escuelas normales públicas del país.

- Programa de Movilidad Académica de Estudiantes de Escuelas Normales en Francia para ofrecer a estudiantes de las escuelas normales públicas del país, experiencias educativas en las universidades de Créteil, Orléans, Poitiers, Bordeaux y Montpellier, mediante la realización de actividades de práctica docente y estudios disciplinarios en campos educativos.

- Proyecto Paulo Freire de Movilidad Académica para Estudiantes de Programas Universitarios de Formación del Profesorado, que tiene el propósito de ofrecer a estudiantes de escuelas normales experiencias educativas en diversos con-textos internacionales de Iberoamérica.

- Programa de Movilidad Académica Nacional de Estudiantes de Escuelas Normales que brinda a los estudiantes de escuelas normales públicas del país, formación en diferentes contextos educativos e institucionales de escuelas normales y universidades públicas, fomentando el intercambio de experiencias didácticas y pedagógicas a nivel interinstitucional.

Otro proyecto importante fue la apertura de procesos de otorgamiento de becas para el perfeccionamiento del idioma inglés en Canadá, del que fueron otorgadas 129 becas a estudiantes de escuelas normales, para realizar la estáncia de estudios por dos meses, durante 2017 2018. Los beneficiarios realizaron los estudios del idioma inglés en el Georgian College, Kaplan International, Lambton College y Trent University.
Ante este escenario, es importante reconocer que la movilidad y cooperación educativa internacional ha tenido un impacto en los procesos de formación de los estudiantes normalistas.

En el caso de la región sur, específicamente Chiapas, los efectos de las políticas educativas han comenzado a surtir efecto en la participación de los alumnos. Sin embargo, en lo que refiere a la participación de alumnos de normales interculturales e indígenas, es tema pendiente; porque las estadísticas muestran que la población de alumnos indígenas que logran ingresar a las escuelas normales es reducida.

Navarrete (2016) sostiene que las posibilidades para estudiantes provenientes de comunidades indígenas que acceden a la educación superior es reducida, y aún menos los que se benefician con los programas de movilidad internacional.

De acuerdo con el Censo de Población y Vivienda (INEGI, 2011), sólo el 5.4\% de los indígenas han realizado estudios universitarios, apenas la mitad del porcentaje registrado de los no indígenas (10\%).

Cabe destacar que uno de los logros de la promulgación de la Ley de los Derechos Lingüísticos de los Pueblos Indígenas (INALI) ha sido el acceso a la educación superior (Del Val, 2017), lo que obligó a las políticas públicas a implementar, con el marco jurídico e institucional los programas de becas para estudiantes indígenas, así como el desarrollo de programas educativos interculturales.

La inclusión de estudiantes indígenas en los programas de movilidad contribuye al proceso de democratización y diversificación que requieren los procesos de internalización de la educación superior. Sin embargo es urgente dar cuenta de las condiciones de igualdad en las que los proyectos se aplica, en especial para los sectores más vulnerables, como los indígenas.

De ahí la importancia de lo que puntualiza García (2013): que la integración económica se ve acompañada por la integración educativa, en donde la movilidad estudiantil desempeña un papel determinante puesto que complementa la formación de los sujetos, impulsa el desarrollo de una identidad regional particular, facilita la integración laboral y cultural, además de posibilitar el contacto con formas de trabajo académico y de expresión cultural diferentes y enriquecedoras; fomenta, también, el intercambio de ideas y de conocimientos, posibilita la realización de proyectos conjuntos que pueden derivar en la formulación de soluciones a problemas 
comunes. Acerca a los ciudadanos de distintos países, con lo que se promueve la construcción de una región con personas más tolerantes y solidarias.

\section{LA EDUCACIÓN INTERCULTURAL, MÁS ALLÁ DE LO CONCEPTUAL}

Al menos tres conceptos centrales giran alrededor del significado que se le atribuye a la educación intercultural: diversidad, interculturalidad y educación.

Se intenta desde la hibridación de los tres hacer una amalgama que permita entender la complejidad del contexto que habitamos y desde ahí procurar otras formas de relación que posibiliten establecer relaciones más horizontales, no sólo entre las personas, sino entre sociedaddes.

Para Nivón (2013) la diversidad parece ser un asunto que a raíz de los movimientos sociales del mundo entero busca garantizar y reivindicar los derechos humanos. El pluralismo, la diversidad y la interculturalidad son valores que orientan el desarrollo de las políticas culturales $y$, en general, de todas las políticas públicas. El reduccionismo de las políticas públicas de atención a la diversidad promovidas desde la lógica positivista de la tolerancia únicamente han acentuado sistemas relacionales de diferenciación. Ante esto, ha resultado imperante las exigencias de los llamados "grupos minoritarios" que en las últimas décadas, no sólo han levantado la voz, sino que han hecho cambios estructurales en todos los sistemas "modernos" establecidos por los grupos de poder.

La diversidad no puede entenderse exclusivamente desde la tolerancia como marca de diferenciación. Tendrá en todo caso, que superar los discursos de la tolerancia, la igualdad y el derecho, establecidos como principios de diferenciación o exclusión social y cultural de los pueblos.

O como Ibáñez Salgado (2004) indica, la diversidad como la consecuencia de modos distintos de construir significados que dan lugar a una visión de mundo diversa en algunos o en muchos sentidos, no mejor o peor sino sólo diferente, según el modo de convivencia propio de cada cultura o subcultura, en el que se destaca el desbordamiento del mono-culturalismo, gracias a las luchas intelectuales-epistémicas que demuestran otras visiones o formas de comprender el mundo.
La diversidad más allá de establecer una sola forma de entender el mundo, diversifica nuestras miradas, no para ser mejores o peores que otros, sino sólo fundar distintas formas de relación desde nuestras diferencias, constitutivas en el lenguaje, según el modo de convivencia propio de cada cultura.

En el ámbito educativo cuando se trata de abordar la diversidad, es común encontrar políticas para la diversidad que utilizan los conceptos como cultural slogan, o en el peor de los casos, como folklore, en el que se implementan propuestas de "inclusión".

Los movimientos sociales alternativos y académicos han puesto en jaque la propia política de Estado que se pronuncia a favor de la diversidad o las propuestas educativas para la diversidad como un discurso de plataforma, que invisibiliza la problemática que de fondo persiste en los contextos sociales y espacios educativos.

En el debate sobre la diversidad lo que se busca es apertura, porque no debe considerarse como el resultado de una construcción social, en tanto que es el contexto social el que determina los modos de diferenciación de las personas: "la diferencia, y por tanto, la forma en cómo se expresa la diversidad, sólo es percibida cuando estamos en un contexto de homogeneidad determinado" (Zapata Barrero, 2010, p. 44).

En efecto, homogeneizar los distintos escenarios de existencia humana ha sido el proyecto más voraz de la modernidad, hacer visible diferencias y a partir de ahí imponer formas y dinámicas de relación que fueron, no sólo condicionando, sino determinando las relaciones entre individuos y de sociedades enteras.

La imposición de estas formas de visión vertical se instituyeron como formas asimétricas de relación, que hasta la fecha siguen imperando en nuestras sociedades. Es necesario reconocer que:

la primera arena no académica en que se discutió el tema de la diversidad, y su definitivo anclaje en el campo político se debió a su conexión con los movimientos sociales: las luchas por los derechos civiles en los Estados Unidos, la realidad de la inmigración en Europa Occidental y el surgimiento de la movilización indígena en Iberoamérica. (Nivón, 2013, p. 28).

En el caso latinoamericano, fue a principios del siglo XXI que "el debate sobre la diversidad adquiere un sentido importante y el pluralismo cultural se ha saldado favorablemente a favor de la incorporación de la diversidad en las políticas 
públicas sociales y de cultura de toda la región" (Nivón, 2013, p. 30).

La discusión se concentró en un despliegue importante de las diferencias de género, orientación sexual o prácticas religiosas que pueden ser instrumentos poderosos para expresar la diferencia que los que se derivan de la nacionalidad o la etnia. Esto último permite comprender el "reconocimiento de todas las formas de diversidad, sean las tradicionales 0 las nuevas expresiones" (Nivón, 2013, p. 33).

La interculturalidad como paradigma adquiere relevancia en un contexto de mayor diversidad, especialmente al presentarse como una propuesta de desmembramiento del paradigma multicultural o mono-cultural que durante mucho tiempo adornó las políticas educativas, programas curriculares en "pro" de la integración o asimilación de los grupos migrantes para el contexto europeo y norteamericano y para la integración de grupos minoritarios en América Latina.

El paradigma intercultural no implica únicamente intercambio o complementariedad, sino conciencia crítica para revisar lo que históricamente subyace como en lo intercultural, entendida ésta como práctica cultural de los contextos propios. A decir de otra manera "impulsar activamente procesos de intercambio que, por medio de mediaciones sociales, políticas y comunicativas, permitan construir espacios de encuentro, diálogo, articulación y asociación entre seres y saberes, sentidos y prácticas, lógicas y racionalidades distintas" (Walsh, 2009, p. 45).

Implica principalmente el reconocimiento del "otro, de los "otros" (individuos, pueblos, culturas, conocimientos, lengua, identidades, etc.) negadas históricamente; es decir, la interculturalidad en el más profundo sentido crítico o autocrítico implica repensar, deconstruir sistemas dominantes y parametrales que se han impuesto históricamente. Es lo que Fornet Betancourt (2004) puntualiza con respecto a los pueblos indígenas. Se requiere de "tarea autocrítica que involucra un diálogo a fondo [...] con sus expresiones históricas, con las instituciones [...], un diálogo con su historia [...], desde sus comienzos, como demuestran, por ejemplo, las luchas hasta hoy ininterrumpidas de los pueblos" (p. 44).

La interculturalidad como categoría conceptual se encuentra en debate, pero no en sentido etimológico, sino en la praxis. En este terreno existe una tensa lucha por aceptar lo que desde las políticas educativas se establece como inter- culturalidad y lo que grupos en resistencia consideran como un proyecto que busca reivindicar las formas de reconocimiento, igualdad y diversidad cultural. Tubino (2004) sostiene que:

la interculturalidad no es un concepto, es una manera de comportarse. No es una categoría teórica, es una propuesta ética. Más que una idea es una actitud, una manera de ser necesaria en un mundo paradójicamente [...] más incomunicado interculturalmente. (p. 155).

Los programas educativos interculturales de formación de profesores, o para la educación básica terminan ajustando lo intercultural a una serie de "principios básicos" como tolerancia, el respeto, los derechos humanos desde los cánones modernos o neocoloniales (Tubino, 2004); que no son más que la continuidad de mantener sistemas de relación asimétrica.

Por eso resulta interesante reconocer, descubrir, dialogar con el otro desde la diferencia, es decir, "no se trata de esencializar identidades o entenderlas como adscripciones étnicas inamovibles" (Walsh, 2009, p. 45).

Más que un discurso, la interculturalidad debe ser una práctica que rompa con sistemas de poder establecidos. Representa ante todo procesos dinámicos bidireccionales, creativos, de ruptura y construcción. Implica desentrañar procesos enraizados históricamente, pero manifiestos en nuestra actualidad "brechas caracterizadas por asuntos de poder y por las grandes desigualdades sociales, políticas y económicas que no permiten relacionarnos equitativamente, y procesos que pretenden desarrollar solidaridades y responsabilidades compartidas" (Walsh, 2009, p. 47).

La educación intercultural como proyecto social, al igual que la interculturalidad como paradigma, tiene un fin último: visibilizar sistemas de relación, ejercicios de poder que subyacen en nuestras acciones como seres humanos; acciones que son producto también de un sistema, de una historia, de un contexto, de ideologías. En suma, de estigmas impuestos sobre nuestras sociedades donde la razón y la verticalidad son común denominador de las relaciones culturales y humanas.

Romper con estos estigmas, implica ampliar el espectro, alzar la mirada y fundar otras formas de relación simétrica que ponga en igualdad, no sólo nuestra condición cultural, sino nuestras más genuinas relaciones humanas. 


\section{METODOLOGÍA}

La investigación se ubica en el enfoque cualitativo, en el que se retoma el enfoque narrativo como método de estudio, principalmente porque interesó resignificar las experiencias de los estudiantes normalistas sobre los proyectos de movilidad y cooperación promovido por la DEGESPE en el periodo 2016-2018.

La investigación de tipo narrativa como dispositivo metodológico posibilita una mirada epistémica, identitaria y constructiva del sujeto que investiga, así como de quienes son investigados, por lo que establece una relación de horizontalidad dialógica que de-construye la forma de hacer investigación.

De este modo se convierte en una propuesta metodológica humanizada que reconoce al otro en su dimensión de sujeto histórico, crítico.

De acuerdo con Goodson (2004) y Bolívar et al. (2001), la investigación narrativa es una modalidad de investigación que nos permite ampliar el conocimiento sobre lo que realmente sucede en la formación académica, a través del punto de vista de los implicados.

Por medio de testimonios (escritos-orales), se recuperan las experiencias en los programas de participación que fortalecen la formación inicial docente. Las orientaciones de investigación narrativa o biográfica narrativa más sobresalientes en el campo de la educación se centran en el ámbito de la formación docente y su profesionalización (Hernández, 2016; Bolívar, et.al., 2001; Álvarez Gayou, 2003).

Las distintas políticas educativas en la última década en México han afectado, por no decir tergiversado la formación docente. Razón de más para el desarrollo de investigaciones que recuperen las experiencias de los profesores, tanto en la formación inicial en las escuelas normales, como en el desarrollo de la profesión. Esto proporciona vías de información a la hora de describir cómo se desarrollan las experiencias formativas en las comunidad académicas.

Para aquellos especialistas, alumnos, profesores que desarrollan investigaciones de tipo biográfico-narrativa se asumen como sujeto en constante transformación, de corresponsabilidad cotidiana, una corresponsabilidad que los implica, no sólo con los resultados, sino con los procesos, las acciones, los sentimientos.

Narrar, recuperar la palabra, las experiencias posibilita la reflexión, al mismo tiempo sentir el acto educativo, es un proceso de interiorizaciónexteriorización, en esa relación con los "otros" que nos acogen y a quienes acogemos con nuestras diferencias, con nuestra humanidad, es de entrada la narración punto de partida para reconocernos, la narración nos identifica en un ámbito eminentemente práctico.

Sin narración no hay, pues, identificación posible ni del individuo ni de las comunidades, "a no ser que toda identificación subjetiva se pierda en la serie episódica de las acciones, haciendo imposible toda identidad subjetiva, o se confine en una ilusión sustancialista que no dará cuenta de la diversidad" (Ricoeur, 2004, pp. 27-28).

La entrevista se realizó con 4 alumnos indígenas de 7 que han participado desde 2016 a 2018 en el Estado de Chiapas, de 4 Escuelas Normales: 1 alumna de la Escuela Normal Primaria "Lic. Manuel Larraínzar", San Cristóbal de Las Casas, 1 alumna de la Escuela Normal Experimental, San Cristóbal de Las Casas, y 2 alumnos de la Escuela Normal Mactumactzá (1 de movilidad internacional y 1 de movilidad nacional), en Tuxtla Gutiérrez. Para la obtención de información se utilizó la entrevista en profundidad para conocer la experiencia de los estudiantes. Como primer momento se solicitó un escrito narrativo sobre la movilidad, posterior a ello se centraron preguntas especificas para ensanchar los argumentos y las historias construidas a partir de la experiencia educativa.

\section{RESULTADOS}

\subsection{ESTADÍSTICAS PREOCUPANTES SOBRE LA COOPERACIÓN Y MOVILIDAD INTERNACIONAL}

En este documento se presentan algunos avances de la investigación que aún se encuentra en aplicación, sin embargo se pueden visibilizar importantes datos que da cuenta del estado que guarda la movilidad y cooperación internacional en la formación de docentes indígenas interculturales.

Al respecto podemos observar que en el concentrado de los proyectos de movilidad la DGESPE (2018) reporta un total de 1.326 participantes en los cuatro programas de cooperación y movilidad nacional e internacional: a) Programa de Capacitación de Directores de Escuelas Normales en Francia, b) Programa de Movilidad Académica de Estudiantes de Escuelas Normales en Francia, c) Proyecto Paulo Freire de Movilidad Académica para Estudiantes de Programas Universitarios de Formación del 
Profesorado, d) Programa de Movilidad Académica Nacional de Estudiantes de Escuelas Normales, de 2015 a 2018, y el programa de otorgamiento de becas para el perfeccionamiento del inglés en Canadá. Los cuales se concentran en el Tabla 1 de la siguiente forma:

Tabla 1. Cooperación nacional e internacional de escuelas normales

\begin{tabular}{|l|c|c|c|c|c|}
\hline \multicolumn{5}{|c|}{ COOPERACION NACIONAL E INTERNACIONAL DE ESCUELAS NORMALES } \\
\hline Programas do cooperación & 2015 & 2016 & 2017 & 2018 & Total \\
\hline Directores en Francia & & 35 & 30 & 50 & 115 \\
\hline Autoridades en Francia & & & 8 & 8 & 16 \\
\hline Docentes en Francia & 3 & & 10 & 13 & 26 \\
\hline Estudiantes en Francia & 29 & 52 & 53 & 50 & 184 \\
\hline Estudiantes España & 96 & & & & 96 \\
\hline Docentes España & 7 & & & & 7 \\
\hline Estudiantes nacional & & 145 & 125 & 157 & 427 \\
\hline Docentes nacional & & & & 43 & 43 \\
\hline Estudiantes en Iberoamérica & & 64 & 36 & $20+63$ & 183 \\
\hline Estudiantes en Canadá & & & 129 & 100 & 229 \\
\hline \multicolumn{1}{|c|}{ Sumas } & 135 & 296 & 391 & 504 & 1,326 \\
\hline
\end{tabular}

Fuente: Estadística de Movilidad DGESPE (2018).

Aunque no se cuentan con los datos de distribución nacional de los Estados y Escuelas Normales participantes, es importante observar el impacto de la estrategia de fortalecimiento que por medio de las convocatorias ha tenido en la participación de los actores educativos de estas instituciones. Al ser una convocatoria abierta se incluyeron normales estatales, federales, públicas, rurales, indígenas e interculturales.

La estadística que corresponde a 19 escuelas normales de Chiapas se ha comportado de la siguiente manera: de un total de 32 participantes en tres años, en 2016 respondieron a las convocatorias nacionales un total de 8 becados; para 2017 se observa un incremento a 13 becarios y en 2018 se reduce el número a 11 becarios, aun que en los tres años se ha participado en la mayoría de los proyectos de movilidad propuestos, entre los que destaca Francia, Iberoamérica, Canadá y la Nacional.
Tabla 2. Movilidad y cooperación internacional en Chiapas

\begin{tabular}{|c|c|c|}
\hline Año & Participantes & Programa \\
\hline \multirow{4}{*}{2016} & 2 directores & Francia \\
\hline & 1 estudiante & Francia \\
\hline & 3 estudiantes & Nacional \\
\hline & 2 Estudiantes & Iberoamérica \\
\hline \multirow[t]{4}{*}{2017} & 2 directores & Francia \\
\hline & 1 estudiante & Francia \\
\hline & 8 estudiantes & Nacional \\
\hline & 2 estudiante & Canadá \\
\hline \multirow[t]{3}{*}{2018} & 1 director & Francia \\
\hline & 5 estudiantes & Nacional \\
\hline & 5 estudiantes & Canadá \\
\hline Total & \multicolumn{2}{|c|}{32 participantes en 3 años } \\
\hline
\end{tabular}

Fuente: Datos DGESPE (2019). Elaboración propia.

Se puede observar que de los 1.326 becarios que han participado en los distintos programas, en Chiapas, de los 32 en la Tabla 2, 27 son estudiantes. En la estadística nacional es poco representativo. Resulta necesario observar en los datos (Tabla 3), que 7 son de origen indígena tsotsil, tseltal y zoque, provenientes de 3 escuelas normales: 5 becarios de la Escuela Normal Rural Mactumactzá, 1 de la Escuela Normal Experimental "Fray M. A. De Córdoba y Ordóñez" y 1 becaria de Esc. Normal "Lic. Manuel Larraínzar".

Tabla 3. Escuelas Normales y alumnos de origen indígena en Chiapas

\begin{tabular}{|c|c|c|c|c|c|}
\hline Institución & $\begin{array}{c}\text { No de } \\
\text { alumnos }\end{array}$ & $\begin{array}{c}\text { Proyecto de } \\
\text { Cooperación/Mo- } \\
\text { vilidad }\end{array}$ & Institución & Año & $\begin{array}{c}\text { Semes- } \\
\text { tre }\end{array}$ \\
\hline $\begin{array}{c}\text { Esc. Normal } \\
\text { Mactu- } \\
\text { mactzá }\end{array}$ & 4 & $\begin{array}{c}\text { b) Programa de } \\
\text { Movilidad } \\
\text { Académica de } \\
\text { Estudiantes de } \\
\text { Escuelas } \\
\text { Normales en } \\
\text { Francia, España y } \\
\text { Canadá }\end{array}$ & $\begin{array}{c}\text { Universidad } \\
\text { de Castilla } \\
\text { La Mancha, } \\
\text { Campus } \\
\text { Cuenca }\end{array}$ & 2017 & 7 7o y 50 \\
& \multicolumn{4}{|c}{} & \\
\hline
\end{tabular}




\begin{tabular}{|c|c|c|c|c|c|}
\hline $\begin{array}{l}\text { Esc. Normal } \\
\text { Mactu- } \\
\text { mactzá } \\
\text { Esc. Normal } \\
\text { Experiment } \\
\text { al "Fray M. } \\
\text { A. De } \\
\text { Córdoba y } \\
\text { Ordóñez" }\end{array}$ & 1 & $\begin{array}{l}\text { d) Programa de } \\
\text { Movilidad } \\
\text { Académica } \\
\text { Nacional de } \\
\text { Estudiantes de } \\
\text { Escuelas } \\
\text { Normales }\end{array}$ & $\begin{array}{c}\text { Benemérita } \\
\text { Escuela } \\
\text { Normal “M. } \\
\text { F. Martínez, } \\
\text { en } \\
\text { Monterrey } \\
\text { Universidad } \\
\text { Autónoma } \\
\text { de Yucatán }\end{array}$ & 2016 & 40 \\
\hline $\begin{array}{l}\text { Esc. Normal } \\
\text { "Lic. Manuel } \\
\text { Larraínzar" }\end{array}$ & 1 & $\begin{array}{l}\text { b) Programa de } \\
\text { Movilidad } \\
\text { Académica de } \\
\text { Estudiantes de } \\
\text { Escuelas } \\
\text { Normales en } \\
\text { Francia, España y } \\
\text { Canadá }\end{array}$ & $\begin{array}{c}\text { Kaplan } \\
\text { Internacion } \\
\text { al English } \\
\text { Toronto, } \\
\text { ON Canadá }\end{array}$ & 2018 & 70 \\
\hline & Total: 7 & & & & \\
\hline
\end{tabular}

Fuente: Datos Escuelas Normales del Estado de Chiapas. Elaboración propia.

De acuerdo con estos resultados, es importante observar que la estrategia de fortalecimiento de las escuelas normales propuestos por la DGESPE, atendiendo a la política de cooperación internacional se está concentrando en la formación inicial del profesorado con programas "in situ, en instituciones interculturales, indígenas o convencionales, o bien en una movilidad hacia países extranjeros [...] en educación intercultural" (Didou, 2013). Aunque de los 5.676 alumnos inscritos entre 2015 2017 en las escuelas normales de Chiapas, la representatividad de alumnos indígenas es seriamente escasa (INNE, 2018).

A nivel nacional se observa que del total de alumnos que asisten a la educación normal en el ciclo escolar 2015-2016 y 2017-2018, sólo el $2.2 \%$ es hablante de una lengua indígena (2.419 estudiantes). Sin embargo, es importante decir que este porcentaje se ha incrementado en los últimos años, pues en el ciclo escolar 20122013 eran 1.5\% (1.958 alumnos) (INEE, 2018). Una problemática pendiente que la política educativa tendrá que considerar como prioridad en la atención a jóvenes indígenas que solicitan el ingreso a las escuelas normales.

Es significativo observar que en los proyectos de cooperación internacional en universidad de la región latinoamericana, se ha aumentado en el número de programas e instituciones destinados exclusiva o estratégicamente a la atención a estudiantes indígenas. Pero se tendrá que hacer mayor énfasis en las escuelas normales como instituciones de educación superior, para inte- grar una mayor participación de alumnos indígenas en los programas.

\subsection{EXPERIENCIAS DE MOVILIDAD Y COOPERACIÓN INTERNACIONAL DESDE LOS ACTORES}

Las primeras impresiones en la experiencia de los jóvenes indígenas, es una mezcla entre sentimientos de incredulidad desencajada con relación a la beca, sin importar si fuera para el extranjero o nacional, un nudo en la garganta les impedía hablar. La mayoría afirman que "salir del pueblo o de la comunidad para venir a estudiar ya era mucho, pero esto es increíble", en efecto para muchos estudiantes indígenas (Vázquez y Hernández, 2014; Czarny, 2015), no solo enfrentan la pobreza, la marginación y discriminación, sino además la falta de oportunidades para continuar estudios en todos los niveles. Las escuelas normales en Chiapas, México, tienen la característica de ingresar alumnos que vienen de las comunidades muy alejadas de la ciudad, quienes solo de ésta manera tienen la oportunidad vivir otras experiencias educativas:

Desde la creación de la escuela normal en 1931 no se había realizado un intercambio académico internacional, me preocupó un poco conocer otro lugar, fue una especie de emoción, nerviosismo, no lo sé [...] algo inexplicable, un sentimiento de alegría que no lograba contener en mi interior [...] ir a España era como algo irreal, y luego me puse a buscar información sobre la Universidad de Castilla La Mancha, me hizo sentir pequeñito por tanta información que encontraba (Movilidad Internacional, Entrevista A. Gómez, 2018).

Soy la primera alumna que participa en intercambio internacional, soy de Chamula e indígena, por eso no me lo creía, estaba emocionada, no lograba entender porque a mí me daban esa oportunidad entre tantos alumnos, pero también mis padres estaban con sentimientos encontrados por esta experiencia (Movilidad Internacional, Zenaida, 2018).

Una característica de estas instituciones, es que el $70 \%$ de los alumnos vienen de comunidades rurales e indígenas, que no siempre reconocen su origen étnico, es decir, existe una negación de la lengua y la cultura desde el ingreso a la escuela normal. Si bien existe una estadística por cada institución a la que deciden ingresar los alumnos, la realidad es que no puntualizan el lugar de origen y el grupo étnico al que pertenecen, lo que no permite tener un porcentaje real de alumnos indígenas en las escuelas normales. 
Sin embargo, durante el proceso de formación en las escuelas normales, los alumnos van construyendo una visión crítica de su identidad, reconociendo el valor de la lengua y la cultura, como afirma Zenaida durante la entrevista: "Soy la primera alumna que participa en intercambio internacional, soy de Chamula e indígena, por eso no me lo creía". También es cierto que este proceso de negación es producto de un proceso de discriminación velada por nuestra propia sociedad con los pueblos indígenas.

En lo que refiere al proceso de acompañamiento administrativo se reconoce que las propias normales en el sur de México al participar en los programas de movilidad y cooperación son novedad, por lo que algunas instituciones redoblaron esfuerzos para abrir los canales de comunicación de apoyo de los alumnos y padres de familia de los alumnos.

Por el primer grupo de alumnos se hizo mucho ruido, en los medios y redes sociales, porque fuimos los primeros en toda la vida de nuestra normal, nos brindaron un buen acompañamiento, los trámites, los papeles y todo lo necesario para estar en tiempo con lo que nos pedía. Existió un buen vínculo con la universidad receptora porque nos brindaron atenciones, apoyo en todas nuestras dudas, eso desde que hicimos contacto por teléfono y por medio de las redes (Movilidad Internacional, Entrevista A. Gómez, 2018).

En efecto, que alumnos de la normal rural se integraran a un programa de movilidad y cooperación internacional, abría nuevas oportunidaddes, no solo para los estudiantes de esa institución, sino que la difusión en los medios de comunicación sobre el programa impactó en la mayoría de las escuelas normales del Estado de Chiapas. Para muchos alumnos, esta fue una importante referencia para participar en próximas convocatorias, en el que puntualizan que: "Después que nuestros compañeros se integraron al programa de movilidad internacional, otros comenzamos a buscar y esperar la beca para [...] (Movilidad Nacional, Entrevista, S. Santiz, 2018). El caso de la Escuela Normal Intercultural, en San Cristóbal de Las Casas, la primer beca de movilidad internacional se otorgó para una alumna indígena de San Juan, Chamula, quien describe su experiencia como un proceso complicado, porque fue el primer intento en la movilidad académica al extranjero.

No tenía a quien preguntarle sobre el procedimiento, tenía muchas dudas de los requisitos de la convocatoria, la cual tuve la necesidad de recurrir a los directivos explicando mis deseos de aplicarle y los motivos, para hacerlo, sin embargo, no se negaron a escucharme, al contrario me orientaron de lo que debía hacer, claro sin ga- rantizarme nada, debido que la selección no hace ella, sino la coordinación de Becas y la DGESPE. (Movilidad Internacional, Zenaida, 2018).

La preocupación no solo se concentró en los procesos administrativos, sino además en las condiciones económicas de los alumnos, que en estos casos, los padres de familia no contaban con los recursos para apoyar a sus hijos. Un aspecto que los alumnos destacaron fue que por fortuna la beca económica se depositó a tiempo, lo que permitió la compra de boletos, renta de los lugares de alojamiento, entre otros retos que enfrentaron los jóvenes indígenas de las escuelas normales.

La experiencia en otros contextos movilizó los procesos de formación, los saberes y proce-sos académicos que viven los estudiantes en las escuelas normales, la diferencia con la universodad o incluso con normales que son del norte del país.

Notaron desigualdad en la misma organización y funcionalidad de las instituciones, el servicio que se les brinda a los estudiantes, las actividades de aprendizajes, el tratamiento en horas teóricas y prácticas, los procesos de evaluación, la intervención y preparación de los docentes que son totalmente distintos. Esto desde el principio preocupó a los alumnos y, posteriormente, les generó problemas en las escuelas normales al incorporarse a sus actividades.

Lo notorio en las experiencias es que, más que cursar materias, la movilidad abre expectativas y reposiciona de forma crítica a los estudiantes, conocer la dinámica de las universidaddes, hace notar lo que más uno afirmó "Las normales en nada se parecen a las universidaddes", ni en su funcionamiento, organización, política y operatividad.

En México, las escuelas normales pasaron de ser instituciones de educación superior como parte de una política de profesionalización, pero en su estructura y funcionalidad siguieron siendo las Escuelas de Educación básica, con una organización administrativa reducida.

De las 19 escuelas normales en Chiapas, el $80 \%$ funciona con una matrícula de entre 90 y 200 alumnos, lo que las mantiene en un sistema de administración centrado en las aulas, no se promueven actividades de gestión, vinculación, como el caso de la movilidad.

Las universidades cuentan con programas propios para la movilidad, mientras que las normales mantienen una organización que depende directamente de la DGESPE. En este caso la movilidad no es un programa permanente, 
sino que depende de la política y administración en turno, por eso afirman los alumnos que:

Las normales en nada se parecen a las universidades, por mucho que se quiera comparar; la universidad donde estuve me demostró lo pequeño que es nuestra institución, pero más que eso, la atención que se brinda a las universidades, la forma en que se nos observa socialmente, las universidades tienen reconocimiento social y en especial en España la universidad tiene mucho prestigio [...] las universidades nos superan en muchas cosas, no me refiero a que sean mejores que nosotros, sino que la atención, las condiciones de infraestructura, el apoyo a los alumnos, el nivel académico de los profesores, las oportunidades que se reciben... un caso específico: para nosotros fue sorpresa ver que las universidades colocan a cientos de alumnos de movilidad, mientras nosotros acá en las normales los pocos se observa como migaja [...] de Chiapas unos cuantos, mientras que de otras universidades la movilidad es constante [...] acá a los normales social y gubernamentalmente se nos considera un problema, se nos observa mal, mientras en otros lugar se nos reconoce por nuestra lucha, por nuestra capacidad de crítica, por no dejarnos de un sistema voraz [...] (Movilidad Internacional, Entrevista A. Gómez, 2018).

El empoderamiento de los jóvenes indígenas en el sur de México en las instituciones de educación superior, y para estos casos de las escuelas normales rurales, está directamente vinculado a las nuevas juventudes, los movimientos sociales y las problemáticas educativas (Vázquez y Hernández, 2014).

Como señala Czarny (2016), la deuda histórica de las universidades latinoamericanas con lo pueblos indígenas y los jóvenes de las comunidades rurales está matizada de desigualdades sociales que desde antaño el sistema educativo no ha logrado resolver.

Al contrario, desde hace más de una década se ha estigmatizado la funciones de los profesores, lo que ha desbordado los movimientos magisteriales en México.

Con ello, la militarización del país, agudizando los problemas como el caso de la desaparición forzada y asesinato de estudiantes, profesores y líderes estudiantiles de escuelas normales rurales.

Otro aspecto en las narraciones de los alumnos es la constante afirmación: "El norte no es el sur, hay diferencias en todo [...] no solo en la infraestructura y el apoyo económico de las normales". En los argumentos de los jóvenes indígenas, destaca lo desigual en las condi- ciones educativas de las instituciones del norte de México:

Aunque somos normalistas todos en este país, estamos con los mismos programas curriculares, lo cierto es que es distinta la formación, la concepción educativa de los compañeros alumnos, incluso de los profesores. [...] las normales del norte del país tienen todo, no necesitan pedir nada, la escuela recibe mucho recurso y apoyo del Estado. En cambio nosotros acá en nuestra gloriosa normal si no lo pedimos, si no marchamos, si no exigimos ni caso nos hace, al contrario año con año observamos cómo se reduce el presupuesto para la educación en Chiapas (Movilidad Nacional, Entrevista, S. Santiz, 2018).

La desaparición forzada de 43 estudiantes en septiembre de 2014 de la escuela normal rural de Ayotzinapa, en el Estado de Guerrero, transformó el escenario político estudiantil del país. En efecto, ese México que los alumnos puntualizan como desigual, con su división social, política y educativa, muestra la realidad de escuelas normales del sur, en el que la pobreza y marginación son notorias.

Para los alumnos estar en escuelas normales y universidades que tienen todas la condiciones, recrudece su mirada crítica:

En Chiapas nos movilizamos [...] lo seguiremos haciendo hasta tener un trato igualitario. Nos movilizamos, no por querer [...] en la normal M. F. Martínez en Monterrey, los alumnos están tranquilos, tienen las condiciones adecuadas, una infraestructura envidiable [...] los alumnos no marchan en las calles, tampoco hacen política, todo se concentra en las clases, las actividades escolares, las prácticas, son muy "obedientes", "compiten" entre ellos para ser los mejores, entregan tareas, están en sus horarios [...]

cada escuela normal es diferente, estar ahí me hizo darme cuenta que sigo reconociendo mi institución por su capacidad académica y política, porque por eso somos mactu [...] hay algo que me llamó la atención al estar en otro lugar: que México es diferente el sur del norte, la desigualdad se siente desde que se llega; mientras en el sur estamos pidiendo aulas o recursos tecnológicos, etc., en el norte la preocupación es otra, que nada tiene que ver con la preocupación económica (Movilidad Nacional, Entrevista, S. Santiz, 2018).

En las experiencias de movilidad, también se reconocen los procesos académicos, especialmente la dinámica y organización de las actividades de producción, gestión, vinculación y difusión del conocimiento, expresiones como: "las normales en nada se parecen con las universodades, estas instituciones están en otro lugar, con otro nivel, o como se dice, otro estatus", no 
por demeritar la función de las escuelas normales en México, sino que precisan aspectos muy específicos, por ejemplo, que toda la actividad académica se reduce a dos procesos específicos, la enseñanza en el aula y las jornadas de prácticas docentes en escuelas primarias.

También destacan la preparación de los académicos, los programas de apoyo, entre otros aspectos en el que se pondera la formación integral.

Estar en la experiencia de movilidad en una universidad en el extranjero, permite reconocer que hace falta promover el conocimiento en las actividades académicas de las escuelas normales en Chiapas, tal como lo sostiene uno de los alumnos: "no tenemos eventos académicos, congresos por ejemplo [...] en la universidad donde estuve, las clases o actividades escolares se combinaban con actividades de difusión, eventos como presentación de libros [...] hay una preocupación por difundir el conocimiento" (Movilidad Internacional, Entrevista A. Gómez, 2018).

Otros aspectos importantes están relacionados con la formación académica, la organización de las actividades escolares.

Sin embargo, es necesario reconocer que en las escuelas normales de Chiapas, el "talón de Aquiles" es la infraestructura tecnológica (hardware y software) para el desarrollo de las actividades académicas, la ausencia del internet, equipos digitales, entre otros. Los alumnos sostienen que desde el primer día de clases observaron la preparación de los profesores, la organización en las actividades, les proporcionaron las planeaciones, la organización de contenidos.

Una de las alumnas puntualiza:

el impacto de una buena enseñanza en Kaplan, por una parte, son los recursos tecnológicos avanzados que cuenta, por ejemplo el pizarrón inteligente (táctil) y por supuesto los servicios importantes tanto para los docentes o los alumnos, el internet, en el salón de clases se pueden usar los buscadores con los celulares, así apoyarnos para entender mejor, por ello, juegan un papel muy importante en el proceso de aprendizaje. [...] me quedé sorprendida que todas las actividades se vinculan con plataformas tecnológicas, yo tengo computadora, así que fue necesario hablar con mis papás para que me enviaran una que compraron a crédito. (Movilidad Internacional, Zenaida, 2018).

Con relación a lo intercultural, las categorías importantes que parecen en la experiencia de los jóvenes, es lo indígena, lo intercultural y lo rural. Por un lado, como proceso de formación y, por otro, como vivencia en las experiencias de movilidad escolar.

En el caso de la formación inicial docente, las escuelas normales rurales e interculturales en México, nacen en distintos momentos, el normalismo rural en su largo aliento de resistencia nacen como escuelas normales regionales y agrícolas desde los años veinte, especialmente para la formación de maestros capacitados para instrumentar la política alfabetizadora gubernamental (Padilla, 2009).

Por su parte las normales interculturales de reciente creación nacen como parte de la oleada discursiva de la interculturalidad de los movimientos sociales en América Latina; de forma coincidente se internan como propuesta educativa para los contextos rurales por un lado, e indígenas por el otro.

Al contar en Chiapas con escuelas normales con estas características, es notorio identificar en los argumentos de los jóvenes indígenas la relación entre educación rural y la educación intercultural con lo comunitario, lo que permite que reflexionen la experiencia de movilidad en contextos donde, ni lo rural, ni lo intercultural se incluyen como parte de la formación profesional, tal como lo señalan:

a la institución donde estuve de movilidad no se tratan temas de ruralismo, tampoco de educación indígena, mucho menos de educación intercultural [...] pero considero que la experiencia me permitió valorar muchos aspectos, entre ellos la identidad cultural, el arraigo cultural con el que nos forman en las escuelas normales. (Movilidad Internacional, Entrevista A. Gómez, 2018).

Sin embargo, la formación como profesores rurales e interculturales representa un recurso importante de interacción, de interaprendizaje, el empoderamiento de los jóvenes indígenas, que se hace visible no como discurso, sino como medio de interacción y convivencia. El reconocimiento de la cultura, de la identidad, el valor de la lengua posibilitan una interacción mucho más abierta.

En palabras de los alumnos se reconoce que su formación está orientado a la atención del pueblo, al rescate de la cultura, al reconocimiento de la lengua:

permitió aprender con los otros, mis iguales, alumnos que en su mayoría establecen una relación de respeto y armonía [...] mi formación en la normal fue determinante para sentirme en igualdad o al mismo nivel con los compañeros de la universidad. (Movilidad Nacional, Entrevista Y. Hernández, 2018). 
Como afirman los jóvenes, no importa si vienes de Chiapas u otro lugar de origen. Lo que observaron, vivieron o sintieron, estrecha el reconocimiento más allá de la cultura propia para la relación e interacción cotidiana, que hay muchos Méxicos, muchos mundos, formas distintas de ser, con multitud de maneras coincidentes de vivir.

La riqueza cultural de nuestro país, solo se puede valorar, en la convivencia, en la interacción, como lo expresa una alumna:

En este intercambio pude vivir en carne propia la interacción cultural, participar en actividades culturales de la institución, en la que se me permitió exponer parte de mi cultura -que en este caso lo hice con dos actividades: baile y gastronomía en el que presenté parte de lo que es mi pueblo y mi cultura-, pero aprendí mucho más de otras culturas que en mi vida hubiera imaginado su existencia. (Movilidad Internacional, Zenaida, 2018).

La experiencia de movilidad permitió a los alumnos, argumentar sobre lo intercultural, no solo como un proceso educativo, sino como convivencia de la otredad, en el que se reconoce el valor de la cultura propia y la distinta: "al interactuar se le da el valor, el respecto y se dignifica de donde vengas o de la cultura que seas, porque en la relación noté una igualdad, nadie intentando imponer una forma de ver el mundo" (Movilidad Internacional, Entrevista A. Gómez, 2018). La movilidad internacional posibilitó una experiencia puntual de convivencia intercultural, en el que es notorio el empoderamiento de los jóvenes indígenas que se forman en las escuelas normales del sur de México.

\section{CONCLUSIONES}

Los resultados aquí expuestos permiten ampliar la mirada con relación a la importancia de la políticas educativas nacionales e internacionales en el país.

Sin embargo se requiere redoblar esfuerzos para fortalecer con más y mejores programas, con mayor cobertura. Que integren cada vez más a estudiantes indígenas de las escuelas normales del sur de México.

La eficiencia, eficacia y calidad de los programas de movilidad y cooperación internacional no solo deben depender de las políticas federales, sino a nivel estatal e institucional. Así, las escuelas normales deben propiciar las condi- ciones para fortalecer los programas con más y mejores servicios.

Las experiencias formativas en otras instituciones de nivel superior nacional o internacional tienen un potente impacto en la formación inicial de profesores rurales e interculturales.

Asimismo, las experiencias de aprendizaje más allá de las actividades académicas favorecen la visión crítica de los alumnos, al visibilizar el empoderamiento que desde las escuelas normales se promueve para fortalecer la conciencia intercultural, la convivencia humana y el interaprendizaje.

\section{REFERENCIAS BIBLIOGRÁFICAS}

- Álvarez Gayou-Jurgenson, J. L. (2003). Orígenes y planteamientos básicos de la investigación cualitativa. Fundamentos y metodología. México: Paidós.

- ANUIES. (2019). Movilidad estudiantil. Recuperado de: http://www.anuies.mx/programas-yproyectos/cooperacion-academica-nacionaleinternacional/cooperacion-academicainternacional/movilidad-estudiantil.

- Arriaga, R., Cabrera, F. y Enríquez, I. (2018). Cartas desde Pátzcuaro. México: CREFAL-DGESPE-OEI

- Barnett, R., (2013). Imagining the University: New Studies in Critical Realism and Education. New York: Routledge. DOI: https://doi.org/10.4324/9780203072103.

- Bolívar, A. Domingo, J.y Fernández, M. (2001). La investigación biográfica narrativa en educación: enfoque y metodología. Madrid: La Muralla.

- Czarny, G. (2016). Jóvenes indígenas y relatos sobre escolaridades en la Universidad Pedagógica Nacional. Revista del Cisen Tramas/Maepova, 4 (1), 137-151.

- Del Val, J. (2017). Estrategias de interculturalidad y educación superior en México. En Mato, D. (Coord.) (2017). Educación superior y pueblos indígenas y Afrodescendientes en América Latina. Políticas y prácticas de inclusión, democratización e interculturalización. Buenos Aires: EDUNTREF.

- DGESPE (2018). Resumen Ejecutivo. México: SEP. Recuperado de:

https://www.gob.mx/cms/uploads/attachment/file/240 634/1.-_Resumen_Ejecutivo__7_.pdf.

- Didou Aupetit, S. (2013). Cooperación internacional y educación superior indígena en América Latina: constitución de un campo de acción. Revista Iberoamericana de Educación Superior (RIES) 11(4), 83-99. DOI:

https://doi.org/10.22201/iisue.20072872e.2013.11.101 
- Fornet Betancourt, R. (2004). Crítica intercultural de la filosofía latinoamericana actual. Madrid: Trotta.

- García, P. J. J. (2013). Movilidad estudiantil internacional y cooperación educativa en el nivel superior de educación. Revista Iberoamericana de Educación, 61, 59-76. Recuperado de: https://rieoei.org/RIE/article/view/600.

- Gómez, H. E. (2014). Decolonizar el desarrollo. Desde la planeación participativa y la interculturalidad en América Latina. Buenos Aires: Espacio. DOI: https://doi.org/10.25100/prts.v0i21.929.

- Goodson, I. (2004). Historias de vida del profesorado. Barcelona: Octaedro.

- Hernández, M. A. (2016). Educación y narrativa. Voces y vivencias de profesores de Chiapas y Oaxaca. México: Universidad Pedagógica Nacional

- Hudzik, J. K. (2015). Comprehensive internationalization: institutional pathways to success. New York, NY: Routledge. DOI: https://doi.org/10.4324/9781315771885.

- Ibáñez Salgado, N. (2004). La interacción prelingüística: primeras coordinaciones de acciones consensuales. Estudios pedagógicos (Valdivia), (30), 61-74. DOI: https://dx.doi.org/10.4067/S071807052004000100004.

- INEE (2018). Panorama Educativo de México 2017. Indicadores del Sistema Educativo Nacional. Educación básica y media superior. Recuperado de: http://publicaciones.inee.edu.mx/buscadorPub/P1/B/1 16/P1B116.pdf.

- INEGI (2011). XIII Censo de Población y Vivienda 2010. Resultado Definitivo, Instituto Nacional de Estadística y Geografía. Recuperado de: https://www.inegi.org.mx/programas/ccpv/2010/.

- Montes, O. J. M. (2018). Cooperación educativa de Escuelas Normales. En $2^{\circ}$ Congreso Nacional de Investigación sobre Educación Normal. Congreso llevado a cabo en México. Recuperado de: http://www.conisen.mx/memorias2018/memorias/6/P6 66.pdf.

- Navarrete, G. D. (2016). La movilidad internacional de estudiantes indígenas.: una mirada desde la experiencia de los becarios del Programa Internacional de becas de Posgrado para indígenas. En $3^{\circ}$ Coloquio Internacional de Educación Superior y Pueblos Indígenas y Afrodescendientes en América Latina. CIESAS. Recuperado de: https://www.rimac.mx/lamovilidad-internacional-de-estudiantes-indigenas-unamirada-desde-las-experiencias-de-los-becarios-delprograma-internacional-de-becas-de-posgrado-paraindigenas/.

- Nivón, B. E. (2013). Las políticas culturales en América latina en el contexto de la diversidad. (pp.23-46). En Grimson, A. y Bidaseca, K.A. (coord.). Hegemonía cultural y políticas de la diferencia. Buenos Aires: CLACSO Recuperado de: http://biblioteca.clacso.edu.ar/clacso/gt/201305131120 51/HegemoniaCultural.pdf.

- Padilla, T. (2009). Las normales rurales: historia y proyecto de nación. El Cotidiano, 154, 85-93.

Recuperado de: http://www.redalyc.org/articulo.oa?id=32512736009.

- Ricoeur, P. (2004). Tiempo y narración. Configuraciones del tiempo en el relato histórico. Tomo I. Buenos Aires: Siglo XXI editores.

- Sebastián, J. (2004). Cooperación e internacionalización de las universidades. Buenos Aires: Biblos.

- SEP-DGESPE (2005). Acuerdo 651 por el que se establece el Plan de estudios para la formación de maestros de educación primaria intercultural bilingüe. Recuperado de:

https://www.sep.gob.mx/work/models/sep1/Resource/ 9721849d-666e-48b7-8433-0eec1247f1ab/a651.pdf.

- Tubino F. (2004). Del interculturalismo funcional al interculturalismo crítico. En Samaniego, M. (comp.), Rostros y fronteras de la identidad (pp. 151-165). Temuco, Chile: Universidad Católica de Temuco.

- Vázquez, G. L.E., y Hernández M. A., (2014). La Juventud, universidad, etnia y profesión: dificultades para permanecer en la educación indígena. Un estudio de caso de los estudiantes de la Licenciatura en Preescolar y Primaria para el medio indígena (Leppemi 90) De La UPN, Subsede San Cristóbal de Las Casas, Chiapas. En el Congreso Nacional de Investigación Educativa, 2013-2014.

- Wadhwa, R. (2016). New Phase of Internationalization of Higher Education and Institutional Change. Higher Education for the Future, 3(2), 227-246. DOI: https://doi.org/10.1177/2347631116650548.

- Walsh, C. (2009). Interculturalidad, estado, sociedad: luchas (de)coloniales de nuestra época. Quito: AbyaYala.

- Zapata Barrero, R. (2010). Diversidad y política cultural. La ciudad como escenario de innovación y de oportunidades. Barcelona: Icaria. 